\title{
Use of Factor $\mathrm{V}$ Leiden genetic testing in practice and impact on management
}

\author{
Anne-Marie Laberge, MD, PhD ${ }^{1,2}$, Bruce M. Psaty, MD, PhD , Lucia A. Hindorff, MPH, PhD , \\ and Wylie Burke, MD, PhD ${ }^{2,5}$
}

\begin{abstract}
Purpose: To assess the use of the genetic test for Factor V Leiden in clinical practice, physician adherence to national and local guidelines, and impacts of test results on patient management. Methods: Chart review of all patients tested for Factor V Leiden during a 1-year period (2003) in a large nonprofit health care system (group health) $(\mathrm{n}=272)$. Results: The test for Factor V Leiden was most often used in nonacute outpatient settings by primary care practitioners, in combination with other tests for procoagulant disorders. Testing was performed more broadly than recommended: $61 \%$ of tests met American College of Medical Genetics guidelines, $46 \%$ of tests met CAP guidelines, and $37 \%$ of tests met group health internal guidelines. The most common rationale for testing was to explain a clinical event (58\%). Patient management was modified more often in heterozygotes $(54 \%)$ than in those with normal results $(13 \%)(P<0.0001)$. Conclusions: The uptake of the test for Factor $\mathrm{V}$ Leiden has not followed existing recommendations. Genetic risk information was used to influence patient management in the absence of supporting evidence related to health outcomes. These results underscore the importance of further research concerning effective prevention and treatment strategies for patients with genetic risk to help translate genetic risk information into improved health outcomes. Genet Med 2009:11(10):750-756.
\end{abstract}

Key Words: Factor V Leiden, genetic test, patient management, clinical practice guidelines, clinical utility

G enetic testing for disease predisposition is perceived as one of the potential benefits of the Human Genome Project. ${ }^{1}$ As Collins et al. ${ }^{2}$ stated in 2003: "Theoretically, the steps by which genetic risk information would lead to improved health are (1) an individual obtains genome-based information about his/her own health risks; (2) the individual uses this information to develop an individualized prevention or treatment plan; (3) the individual implements that plan; (4) this leads to improved health;

From the ${ }^{1}$ Institute for Public Health Genetics; ${ }^{2}$ Center for Genomics and Healthcare Equality, ${ }^{3}$ Departments of Medicine, Epidemiology, and Health Services, Cardiovascular Health Research Unit, University of Washington, Seattle, Washington; Center for Health Studies, Group Health, Seattle, Washington; ${ }^{4}$ Department of Epidemiology, Cardiovascular Health Research Unit; and ${ }^{5}$ Department of Medical History and Ethics, University of Washington, Seattle, Washington

Anne-Marie Laberge, MD, PhD, Service de génétique médicale, 6e étage bloc 7, CHU Sainte-Justine, 3175 Cote-Ste-Catherine, Montreal, Quebec, Canada H3T 1C5. E-mail: anne-marie.laberge.hsj@ssss.gouv.qc.ca.

Anne-Marie Laberge is currently at Medical Genetics Division, Department of Pediatrics, CHU Sainte-Justine, Montreal, Quebec, Canada. Lucia A. Hindorff is currently at the National Human Genome Research Institute, National Institutes of Health, Bethesda, MD.

Disclosure: The authors declare no conflict of interest

Submitted for publication March 6, 2009.

Accepted for publication June 19, 2009.

Published online ahead of print August 6, 2009

DOI: 10.1097/GIM.0b013e3181b3a697 and (5) health care costs are reduced." However, few genetic susceptibility tests are clinically available and little is known about the acceptance of this practice model. As the authors themselves acknowledge, "scrutiny of these assumptions is needed."

Factor V Leiden (FVL), a common genetic variant, offers an opportunity to study physicians' use of a genetic susceptibility test. Five to $7 \%$ of individuals of European descent and up to $2 \%$ of individuals in other ethnic groups are heterozygous for FVL. The FVL confers a 3- to 4-fold increased risk of occurrence of venous thromboembolism ${ }^{3-5}$ and an increased risk of recurrent pregnancy loss. ${ }^{6,7}$ The FVL may also play a role in arterial thrombosis, pre-eclampsia, placental abruption, and intrauterine growth retardation but data were inconsistent. ${ }^{5,8}$ The American College of Medical Genetics (ACMG) and the College of American Pathologists have issued practice guidelines to define clinical indications for test use. ${ }^{9-11}$

This study describes the use of the genetic test for FVL in a large health system (Group Health [GH] of Puget Sound). We were interested in the circumstances of test use, the use of genetic counseling, and whether the physicians use test results to modify patient management.

\section{METHODS}

\section{Study population}

All patients who had a FVL test in 2003, as recorded in the GH laboratory database, were eligible. There were 273 eligible individuals. One was excluded because his medical chart was physically unavailable. We did not pick a more recent time period for practical reasons: electronic medical records (EMRs) were implemented at GH starting in 2004 and Health Insurance Portability and Accountability Act (HIPAA) regulations prevented us from doing the study in EMRs (2004 and after). The research team was not employed by the GH. Access to the EMR could not be restricted to only the relevant records under study. Given the potential for HIPAA violations in an unrestricted environment, access to the EMR was not available to the researchers. We consider that these 2003 data are representative of current test use because the genetic test for FVL has been available at GH since 1995 and had been routinely used for years by 2003. Furthermore, in 2003, clinical practice guidelines from the College of American Pathologists (2001) and the American College of Medical Geneticists (2002) were already available as were internal GH guidelines (2002). ${ }^{9,10,12}$ Both the ACMG and internal GH guidelines have since been updated (in 2005 and 2003, respectively), but FVL testing indications have remained the same. ${ }^{11,13}$

\section{Variables}

We reviewed the medical charts of patients who were tested for FVL by GH during a 1-year period (2003 calendar year). Information was collected about the context of testing including physician characteristics, patient characteristics, and characteristics of the clinical situation in which testing was performed. Any pretest or posttest counseling about the genetics of FVL, 
the risk for family members, or the potential for insurance discrimination was noted. Changes in patient management after obtaining the test result and reasons for making said changes were noted in notes from follow-up visits, including changes in length or type of treatment or prophylaxis and changes in recommendations about other risk factors (for instance, surgery or immobilization) or medications (oral contraceptives and hormone replacement therapy). A project manager at Group Health's Center for Health Studies used the GH EMRs to provide missing information from the outpatient paper record for 53 patients. Access to these records by this $\mathrm{GH}$ employee insured full compliance with HIPAA regulations and allowed for more complete data ascertainment. The Group Health $\mathrm{Hu}-$ man Subjects Review Committee approved the study.

\section{Data collection and analysis}

Data collection was performed between January and June 2007. A single reviewer (A.M.L.) used a data extraction sheet approved by the Group Health Human Subjects Review Committee to extract all relevant data from the medical records. Data were entered in a database and coded for subsequent analysis.

To accurately identify the physician's rationale for using the test, photocopies of the relevant note in the medical record were made for subsequent coding. These copies were anonymized: all patient and physician identifiers were blacked out. Using the anonymized notes, two different coders (AML, WB) identified and coded the physician's rationale for testing. A priori, we hypothesized that there were four different rationales for FVL testing: (1) explain a clinical event; (2) estimate the risk of recurrence of VT; (3) direct clinical management; and/or (4) facilitate family-based detection of others at risk. During the analysis, the rationale for testing in each case was identified and coded as one or more of the above categories. Cases where no rationale could be identified were coded "unclear/unknown." If the rationale did not fit in any of these categories, it was coded as "other" and added to the list. After a first round of coding, the definition of each rationale was clarified. Other identified rationales found to be frequent ( $>5 \%$ of individuals) were added to the existing list and rationale found to be rare $(<1 \%)$ were dropped. The second round of coding was performed for all charts using the following rationales: (1) explain a clinical event; (2) direct clinical management; (3) because of a family history of VTE, FVL, or both; (4) because of patient request; (5) because of patient anxiety; and (6) unclear/unknown. Interrater agreement was assessed with kappa statistics, which were computed for each rationale.

We assessed the relationship between the test result (positive [heterozygote and homozygote] or negative) and changes in patient management. Types of changes in patient management were described in the Variables section. Change was coded as: (1) present (physician mentions having made a change in patient management); (2) absent but considered (physician mentions a possible change but does not implement it, e.g., "no need to increase current dose of anticoagulation treatment"), (3) absent (physicians does not change patient management, including specifying that no change is made), or (4) unknown (physician note is illegible or does not contain information about patient management). Because current clinical guidelines do not support changes in patient management based on the FVL status, the null hypothesis was that physicians did not change patient management based on the FVL test results. Statistical analysis was performed using Intercooled Stata 8 . Impact on patient management was compared across genotypes using a $\chi^{2}$ test for independence, to look for departures from expected distribution. We also assessed stated reasons for changes in management. Reasons were coded as (1) only due to FVL status (only factor mentioned to support change), (2) in part due to FVL status (FVL status mentioned in combination with other reasons), (3) not due to FVL status (other reasons mentioned to support change, but FVL not mentioned or explicitly excluded as a reasons for change), and (4) unknown (no reason given). Because of small numbers, a two-tailed Fisher exact test was used to compare the reason for management modification across genotypes, to look for departures from expected distribution.

\section{Criteria for adherence to guidelines}

For each test, adherence to relevant clinical practice guidelines for testing was assessed and coded as a dichotomous variable (adherence versus nonadherence). Two professional clinical practice guidelines available in 2003 provided recommendations regarding whom to test. They were issued by the ACMG (2001) and the College of American Pathologists (2002). ${ }^{9,10}$ Internal GH practice guidelines also addressed who should be tested for FVL in the context of VTE. ${ }^{12}$ The criteria used to determine adherence to guidelines are summarized in Table 1. A patient was determined to have been tested in agreement with the guidelines if he/she met at least one criterion for testing, whether the physician explicitly listed the testing indication. For each set of guidelines, the null hypothesis was that physicians use FVL testing in accordance with the guidelines that is physicians would only consider and perform testing for individuals who fit the test indications listed in the guidelines.

\section{RESULTS}

\section{Demographics}

The mean age of the 272 individuals tested for FVL at GH in 2003 was 48.0 years $(95 \% \mathrm{CI}=46.1-49.9$ years $)$. The female: male ratio was 1.92:1. The test identified 48 heterozygotes $(17.6 \%)$ and 1 homozygote $(0.4 \%)$. Of the 272 individuals tested for FVL in 2003, 4.8\% of individuals had previously been tested for FVL and 2.6\% were tested twice during 2003.

\section{Clinical setting}

Testing took place during a regular outpatient visit in most cases (79.8\%), but was occasionally performed in the emergency department $(3.3 \%)$ or during inpatient care $(9.6 \%)$. Family practitioners were the biggest users of the FVL test, accounting for $41.5 \%$ of the tests performed. Other users include internists (14.3\%), obstetricians $(12.1 \%)$, hemato-oncologists $(7.4 \%)$, neurologists $(6.6 \%)$, and pulmonologists (3.3\%). Nonphysicians (midwives and physician assistants) performed $5.5 \%$ of the tests.

Testing was performed for FVL alone in $19.1 \%$ and otherwise was performed in combination with other thrombophilia tests, including anticardiolipin antibodies (57.0\% of 272$)$, protein $\mathrm{C}$ and protein $\mathrm{S}$ levels (50.4\%), antithrombin III levels (43.4\%), presence of lupus anticoagulant $(42.3 \%)$, presence of prothrombin G20211A mutation (29.0\%), and presence of MTHFR C677T polymorphism (19.1\%). Only $1.5 \%$ of patients were tested for all seven additional tests. The median number of tests performed with FVL was 3 (range $0-7$ ).

On the basis of the content of chart notes, none of the patients received formal counseling from a genetics professional. Before testing, physicians documented having provided counseling about the genetic nature of FVL or the potential risk to family members in their chart notes only in $0.7 \%(n=2)$ of patients; counseling was documented in $3.3 \%$ after the test results were available. Of the 49 patients with positive test results (heterozygotes and homozygote), posttest counseling about the genetic nature of FVL or the potential risk to family members from their 
Table 1 Criteria for adherence to guidelines

\begin{tabular}{|c|c|c|c|}
\hline & ACMG 2001 & CAP 2002 & GHC 2002 \\
\hline \multirow[t]{4}{*}{ First episode of VTE } & Age younger than $50 \mathrm{yr}$ & Age younger than $50 \mathrm{yr}$ & $\begin{array}{l}\text { Age younger than } 50 \mathrm{yr} \text { and } \\
\text { idiopathic }\end{array}$ \\
\hline & Any age if family history of VTE & Any age, unprovoked & $\begin{array}{l}\text { Age younger than } 50 \mathrm{yr} \text { and } \\
\text { strong family history of DVT }\end{array}$ \\
\hline & If pregnant or taking o.c. & $\begin{array}{l}\text { Any age, if family history of VTE } \\
\text { at age younger than } 50 \mathrm{yr}\end{array}$ & If pregnant \\
\hline & $\begin{array}{l}\text { Age older than } 50 \mathrm{yr} \text { if no } \\
\text { malignancy }\end{array}$ & $\begin{array}{l}\text { If pregnant, postpartum, taking } \\
\text { o.c. or HRT }\end{array}$ & \\
\hline Recurrent VTE & Yes & Yes & $\begin{array}{l}\text { Same criteria as first episode } \\
\text { of VTE }\end{array}$ \\
\hline VTE at unusual site & Yes & Yes & $\begin{array}{l}\text { Same criteria as first episode } \\
\text { of VTE }\end{array}$ \\
\hline $\begin{array}{l}\text { Pregnancy outcome (other than } \\
\text { fetal loss) }\end{array}$ & $\begin{array}{l}\text { Unexplained severe pre-eclampsia, } \\
\text { IUGR, or placental abruption }\end{array}$ & No & NA \\
\hline Fetal loss & If recurrent or stillbirth & $\begin{array}{l}\text { If unexplained and in second or } \\
\text { third trimester }\end{array}$ & NA \\
\hline $\begin{array}{l}\text { Arterial thrombosis } \\
\text { (including stroke) }\end{array}$ & $\begin{array}{l}\text { No (except if age younger than } 50 \\
\text { yr and no other risk factors) }\end{array}$ & $\begin{array}{l}\text { No (except if unexplained and no } \\
\text { risk factors or in young smokers) }\end{array}$ & NA \\
\hline Myocardial infarction & Females if age younger than $50 \mathrm{yr}$ & No & NA \\
\hline Family history of FVL & Yes & Yes & Only if first-degree relatives \\
\hline Family history of VTE & If VTE at age younger than $50 \mathrm{yr}$ & No & No \\
\hline
\end{tabular}

physician was documented in only $10.2 \%$, including the only homozygote. Among the 223 patients with negative results, posttest counseling was documented in $1.8 \%$. There was no documented discussion of the potential risk of genetic discrimination in insurance.
Testing indications are defined in terms of patient characteristics such as age, sex, type of event they experienced, or circumstances of that event (Table 2). We identified nine indications for FVL testing in this group of patients. The most common testing indication was a first event of VTE $(26.5 \%)$.

Table 2 Adherence to clinical practice guidelines by testing indication ${ }^{a}$

\begin{tabular}{|c|c|c|c|c|}
\hline Indication & $\begin{array}{l}\text { Number of patients } \\
\text { tested for this indication (\%) }\end{array}$ & ACMG & CAP & $\mathrm{GHC}$ \\
\hline First event of VTE & $72 / 272(26.5)$ & $63 / 72$ & $40 / 72$ & $17 / 72$ \\
\hline Recurrent event of VTE & $32 / 272(11.8)$ & $32 / 32$ & $32 / 32$ & $10 / 32$ \\
\hline VTE at unusual site & $9 / 272(3.3)$ & $7 / 9$ & $7 / 9$ & $2 / 9$ \\
\hline Abnormal pregnancy outcome (excluding fetal loss) & $10 / 272(3.7)$ & $9 / 10$ & $3 / 10$ & NA \\
\hline Fetal loss & $25 / 272(9.2)$ & $24 / 25$ & $9 / 25$ & NA \\
\hline Arterial thrombosis (including stroke) & $40 / 272(14.7)$ & $3 / 40$ & $4 / 40$ & NA \\
\hline Myocardial infarction & $2 / 272(0.7)$ & $1 / 2$ & $1 / 2$ & NA \\
\hline Family history of VTE & $38 / 272^{b}(14.0)$ & $31 / 38$ & $21 / 38$ & $11 / 38$ \\
\hline Family history of FVL & $36 / 272(13.2)$ & $36 / 36$ & $36 / 36$ & $30 / 36$ \\
\hline Overall & & $61.0 \%(166 / 272)$ & $45.6 \%(124 / 272)$ & $37.2 \%(58 / 156)^{c}$ \\
\hline \multicolumn{5}{|c|}{$\begin{array}{l}{ }^{a} \text { Testing indications are not mutually exclusive. } \\
{ }^{b} \text { Fifteen were also tested for first episode of VTE, three for recurrent VTE, and six for family history of FVL. } \\
{ }^{c} \text { Total for adherence to GHC guidelines only applies to patients tested in the context of VTE or family history of VTE/FVL because the guidelines were limited to those } \\
\text { indications. } \\
\text { NA, not applicable. }\end{array}$} \\
\hline
\end{tabular}


Patients were also tested for recurrent VTE (11.8\%) and VTE at an unusual site $(3.3 \%)$. Arterial thromboses were the reason for testing in $14.7 \%$ and myocardial infarction accounted for $0.7 \%$. Among women, fetal loss $(9.2 \%$ of total; $14.0 \%$ of women) and other abnormal pregnancy outcomes $(3.7 \%$ of total; $5.6 \%$ of women) were other common indications. A total of $12.1 \%$ had other indications (priapism, epistaxis, thrombosis of arteriovenous fistula, etc.). Of those tested in relation to a clinical event, $14.3 \%$ were tested for FVL before the diagnosis was confirmed.

A family history of FVL, VTE, or both was the only test indication in $15.8 \%(11.0 \% \mathrm{FVL}, 2.9 \% \mathrm{VTE}$, and $1.9 \%$ both). A family history of FVL was used as an indication for testing in combination with a personal clinical event in only one individual, whereas testing in the setting of a family history of VTE in combination with clinical events occurred in $9.2 \%$. Finally, the medical record provided no obvious clinical indication for FVL testing in $3.3 \%$.

\section{Rationale for testing}

The rationale for testing is the physician's motivation for requesting the test. It is related to the clinical utility of the test, i.e., how the test result will be used in patient care. The most common rationale behind FVL testing was to explain a clinical event ( $n=153,58 \%$ ) followed by having a family history of VTE, FVL, or both $(n=48,18 \%)$ and to direct clinical management ( $n=31,12 \%$ ) (Table 3 ). No rationale was identified in $10 \%$ of cases.

\section{Adherence to guidelines}

Adherence to guidelines was highest for the ACMG guidelines: $61 \%$ of patients were tested in accordance with the testing indications found in this guideline (Table 2). Adherence was lower with the CAP guidelines $(45.6 \%)$ and the $\mathrm{GH}$ guidelines $(37.2 \%)$

\section{Impact on patient management}

Overall, physicians modified clinical management for only $20.2 \%$ of patients. Changes in patient management included changes in length or type of anticoagulation treatment or prophylaxis ( 1 of 1 homozygote, 14 of 26 heterozygotes, and 20 of

\begin{tabular}{lccc}
\hline Table 3 Rationales for testing & \\
\hline & $\begin{array}{c}\text { Observed } \\
\text { interrater } \\
\text { agreement } \\
(\%)\end{array}$ & Kappa & $\begin{array}{c}\text { Number of cases for which } \\
\text { both observers agreed that } \\
\text { this rationale was present } \\
(\%)\end{array}$ \\
\hline $\begin{array}{l}\text { To explain a clinical } \\
\text { event }\end{array}$ & 88 & 0.74 & $153(58)$ \\
$\begin{array}{l}\text { To direct clinical } \\
\text { management }\end{array}$ & 89 & 0.61 & $31(12)$ \\
$\begin{array}{l}\text { Because of family } \\
\text { history of VTE, }\end{array}$ & 91 & 0.75 & $48(18)$ \\
$\quad \begin{array}{l}\text { FVL, or both } \\
\text { Because of patient } \\
\text { request }\end{array}$ & 94 & 0.67 & $19(7)$ \\
$\begin{array}{l}\text { Because of patient } \\
\text { anxiety }\end{array}$ & 96 & 0.55 & $8(3)$ \\
\begin{tabular}{l} 
Unclear/unknown \\
\hline
\end{tabular} & 91 & 0.64 & $27(10)$
\end{tabular}

Table 4 Impact of testing on patient management by FVL status

\begin{tabular}{lccr}
\hline Modification & Heterozygote & Normal & Total \\
\hline Yes & 26 & 28 & 54 \\
No, but considered & 6 & 32 & 38 \\
No & 15 & 150 & 165 \\
Unknown & 1 & 13 & 14 \\
Total & 48 & 223 & 271 \\
\hline Chi-square: $P<0.0001$ (with and without "unknown"). & & \\
\hline
\end{tabular}

Table 5 Basis for modification of patient management by FVL status

\begin{tabular}{lccc}
\hline Basis & Heterozygote & Normal & Total \\
\hline Only FVL status & 15 & 1 & 16 \\
In part FVL status & 8 & 5 & 13 \\
Not FVL status & 1 & 21 & 22 \\
Unknown & 2 & 1 & 3 \\
Total & 26 & 28 & 54 \\
\hline
\end{tabular}

Fisher exact test: $P<0.0001$ (with and without "unknown").

28 normal), changes in recommendations about the use of other medications - mostly oral contraceptives and hormone replacement therapy - ( 8 of 26 heterozygotes and 5 of 28 normal), or changes in recommendations about other risk factors, such as surgeries or long-distance travel (1 of 1 homozygote, 10 of 26 heterozygotes, and 3 of 28 normal).

The proportion of individuals for whom management was modified was higher in heterozygotes (54\%) than normal individuals (13\%) (Table 4). The decision to modify patient management was associated with FVL status $\left(\chi^{2}: P<0.0001\right.$, with/without individuals with unknown impact on management). On the basis of the FVL status alone, physicians were more likely to modify patient management in individuals found to be heterozygote for FVL (15 of 26) than in normal individuals ( 1 of 28) (Table 5). The basis for modification of patient management was associated with FVL status (Fisher exact test: $P<0.0001)$. The only homozygote was excluded from statistical analysis.

\section{DISCUSSION}

A majority of FVL tests in this study were requested by primary care practitioners, performed in nonacute outpatient settings, and performed in combination with tests for other procoagulant disorders. Testing was performed more broadly than the testing indications recommended by available clinical practice guidelines. Pretest counseling was documented in only 2 of the 272 individuals tested and posttest counseling for only a minority of the positive cases (heterozygotes and homozygote). The rationale for testing was predominantly to explain a clinical event. Patient management was modified more often in those identified as heterozygotes than in those with normal results. 
As discussed in the Methods section, we chose 2003 as our observation period because of electronic record access restrictions imposed by HIPAA regulations. We have made the assumption that test use has not changed significantly between 2003 and 2007 because FVL testing is as readily available now as it was then, and practice guidelines were available before 2003 and testing indications have remained the same since then. ${ }^{9-13}$ Even in the case that test use has changed since then, this work is still a good example of the use of genetic susceptibility test by physicians in practice and how it compares with recommended test use found in recently issued professional clinical practice guidelines.

Our method of medical chart reviews has certain limitations. Because we defined our population as all individuals who had been tested for FVL, we do not have any information about individuals who would have been eligible for testing according to the guidelines but who were not tested. For our selected population, data collection was limited to the information available in the patient's medical record. Some information was always present and easy to identify (e.g., age and sex), but the availability of other types of information depended on the level of detail provided in physicians' notes. Physicians may not necessarily include information about their decision-making process or the content of their conversation with the patient. The rationale for testing was unclear or unknown in $10 \%$ of patient notes. Even when some information was provided, the rationale was not always made clear. This explains our imperfect interobserver agreement rates. It also led us to redefine some of our a priori rationales. For example, on the first round of coding, we hypothesized that a reason for testing would be "to facilitate family-based detection of others at risk." We changed this to "because of family history" in the second round of coding, because physicians did not clearly indicate whether testing individuals with a family history was done to estimate the risk of future events, to direct management in terms of this potentially increased risk (treatment, prevention, etc.), or to facilitate the identification of other family members at risk. Differences can also be found in the level of detail given about patient management. They may be more likely to be documented in individuals with positive test results (heterozygotes and homozygotes). Although changes in patient management-e.g., use of medications and length of treatment - are likely to be documented, changes that were considered but decided against wont necessarily be documented in the chart. For this reason, it is possible that we underestimated some measures, such as the proportion of individuals who received genetic counseling or for whom treatment modifications were considered but not implemented. Also, the selection of a population of patients who had been tested for FVL did not allow us to determine the likelihood of FVL testing for a particular clinical indication.

Conversely, the chart review allowed us to describe the actual use of FVL testing in practice. By basing our study in a large nonprofit health care system, we were able to assess test use in outpatient, emergency, and inpatient settings. Our results show that FVL testing is performed more often in elective cases in outpatient settings than could be expected. Because my findings are limited to $\mathrm{GH}$, I cannot determine if this finding applies to other settings.

Our decision to study all individuals tested for FVL allowed us to describe the broad range of indications for which the test is used. In addition, although medical records may provide incomplete documentation, they provided information about patient requests and physician decision making that is not available in administrative databases.

The DNA-based test to identify FVL has become one of the first genetic susceptibility tests to be routinely used in practice. The GeneTests directory lists 68 different laboratories in the United States offering clinical testing for FVL, more than for other commonly used genetic tests for cystic fibrosis (55 laboratories) or hemochromatosis (38 laboratories). ${ }^{14}$ Our findings suggest that the uptake of FVL testing does not necessarily follow available recommendations or evidence and is in keeping with other data on FVL use. For example, a survey performed in Canada asked obstetricians to indicate their management recommendations for five clinical scenarios involving pregnant women with FVL and various clinical presentations. ${ }^{15}$ The survey showed that physicians tended to favor giving anticoagulation therapy to pregnant women with FVL to prevent the occurrence of VTE, despite a lack of scientific evidence to support such decisions.

The ACMG and CAP guidelines focus on testing indications and not on patient management. The ACMG guidelines acknowledge that "identification of FVL heterozygosity does not change the therapeutic approach to venous thrombosis or subsequent prophylaxis in most patients," whereas the CAP guidelines state that "there is currently no evidence that the acute management of venous thromboembolic events (length or strength of anticoagulation) should be different in patients with inherited thrombophilia." 9,10 The testing indications found in ACMG and CAP were less restrictive than GH guidelines. This might explain why adherence to guidelines was greater for the professional guidelines than for the $\mathrm{GH}$ internal guidelines. Conversely, GH physicians were more likely to be aware of internal guidelines, making the overuse of the test all the more surprising in the face of such restrictive guidelines. Alternatively, low adherence may be an indicator that physicians are unaware of or insufficiently acquainted with all existing guidelines and clinical evidence. We have no way of knowing if physicians were knowingly choosing not to adhere to guidelines, but our observations identified both overuse and underuse of testing, which suggest that physician decision making is influenced by other factors. As new genetic susceptibility tests become available, it is important to understand that appropriate use of tests will likely require more than the availability of guidelines.

Low compliance to guidelines has been observed in other settings. There is limited evidence on the effectiveness and efficiency of guideline dissemination and implementation strategies. ${ }^{16}$ The issuance of guidelines by professional organizations is not sufficient to motivate their uptake in practice: strategies must be in place to disseminate them to the appropriate physician audience and to facilitate their implementation in practice. For example, a national survey of colonoscopic practitioners in the United Kingdom has shown that only $17 \%$ of them used the most commonly followed guidelines accurately, whereas $69 \%$ made incorrect surveillance recommendations. ${ }^{17}$ This study, like ours, highlights the fact that widespread lack of awareness or familiarity with guidelines can potentially lead to misuse or overuse of resources and that guidelines should include strategies to facilitate their implementation in practice.

Our results show that FVL testing is mostly performed electively in an outpatient setting. During our observation period, electronic records were not available. This might explain why $4.8 \%$ of patients were tested more than once for FVL (and $2.6 \%$ within 2003) if the previous test result was not readily accessible in the paper record. We hope that access to patients' EMRs will have reduced such unnecessary testing, but a recent study 
supports our observation and suggests that duplicate testing is an ongoing problem: duplicate testing rates within a 1-year period were $0.3 \%$ for HFE and $3.3 \%$ for TPMT and reached $1.9 \%$ and $6.9 \%$, respectively for all-time incidence of duplicate testing. ${ }^{18}$

The mostly elective nature of FVL testing fits well with the first step of Collins' model: "1) an individual obtains genomebased information about his/her own health risks." (2) We found that the most common rationale for testing was to explain a clinical event, but the presence of FVL is neither diagnostic of an event nor necessarily its only etiologic factor. We can hypothesize that physicians hope to direct clinical management once they identify etiologic or contributing factors, but did not find evidence of this kind of decision-making plan in patients' charts. It is possible that physicians decide to perform testing because the test result provides information about the context in which the event has occurred. In that case, the test result is one of many elements, contributing to the patient's clinical situation. On the basis of the patient's clinical situation as a whole, the physician might decide to modify patient management but would not attribute this modification directly to the FVL test result. The absence of a clear link between test result and a clinical response meant to improve health outcomes precludes the ability of patients and physicians to undertake the second step of Collins" model: "2) the individual uses this information to develop an individualized prevention or treatment plan." Furthermore, our data show that patients were rarely counseled about the risk to family members, thereby preventing at-risk relatives from gaining relevant information to develop their own prevention plans.

A number of interventions have been suggested for individuals heterozygote for FVL, including prolonged anticoagulation therapy after a VTE event, prophylactic anticoagulation in certain risk situations (for instance, pregnancy), and avoidance of certain drugs (for instance, oral contraceptives and hormone replacement therapy). ${ }^{5}$ Lifestyle modifications have also been suggested, for instance, avoidance of nongenetic risks where possible or specific measures to decrease risk, such as leg exercises during long airplane flights. However, the effectiveness (and cost effectiveness) of these interventions is still under investigation. The current evidence does not suggest that changes in treatment or surveillance after the identification of FVL status in a given individual are likely to improve the individual's health outcomes. For example, a recent systematic review has concluded that there were insufficient data to demonstrate the effectiveness of prophylactic anticoagulation therapy in pregnant women with thrombophilia and the relative effectiveness of prophylaxis in individuals undergoing orthopedic surgery. ${ }^{5}$ Although our study did not look at long-term health outcomes, the fact that physicians are currently modifying patient management based on genetic risk information in the absence of evidence to support such changes was worrisome, especially considering that anticoagulant therapy and prophylaxis have known risks of bleeding. ${ }^{19}$

The availability of genetic risk information will only lead to improved health outcomes if the second step of Collins' theoretical model can be achieved. Improved health outcomes can ultimately be achieved only if individuals and their physicians can choose from evidence-based preventive strategies or treatments to devise individualized management plans. Further research is needed to determine how the genetic risk information provided by FVL can be used to improve health outcomes. ${ }^{20}$ Such evidence will also be needed for any new genetic susceptibility test. Practice guidelines that provided indications for testing could have encouraged test use in our study sample; an increase in test use in response to new clinical practice guidelines has been documented for other genetic tests, like cystic fibrosis carrier screening or first trimester screening for Down syndrome. ${ }^{21,22}$ However, actual use exceeded the indications specified by the guidelines in our case. Our results suggest that genetic susceptibility tests may be taken up by physicians and patients, especially in the presence of guidelines, even if they are introduced before effective prevention and treatment strategies are available or in the absence of clear evidence for test-based clinical management. Educational strategies are needed to inform physicians and patients about the appropriate use of genetic risk information based on the currently available evidence.

\section{ACKNOWLEDGMENTS}

This work was supported, in part, by Grant P50HG003374 from the National Human Genome Research Institute and the National Institute for Child Health and Human Development (Burke) and, in part, by Grants HL74745, HL087652, and HL085251 from the National Heart, Lung, and Blood Institute (Psaty). Anne-Marie Laberge is a recipient of a doctoral training award from the Fonds de Recherche en Santé du Québec (FRSQ).

The authors thank Helene Starks for revising the manuscript for clarity. Anne-Marie Laberge had full access to all of the data in the study and takes responsibility for the integrity of the data and the accuracy of the data analysis. The funding organizations and sponsors had no role in the design and conduct of the study; collection, management, analysis, and interpretation of the data; or in the preparation, review, and approval of the manuscript.

\section{REFERENCES}

1. Collins FS, McKusick VA. Implications of the Human Genome Project for Medical Science. JAMA 2001;285:540-544.

2. Collins FS, Green ED, Guttmacher AE, Guyer MS. A vision for the future of genomics research. Nature 2003;422:835-847.

3. Emmerich J, Rosendaal FR, Cattaneo M, et al. Combined effect of factor V Leiden and prothrombin 20210A on the risk of venous thromboembolismpooled analysis of 8 case-control studies including 2310 cases and 3204 controls. Study Group for Pooled-Analysis in Venous Thromboembolism. Thromb Haemost 2001;86:809-816.

4. Langlois NJ, Wells PS. Risk of venous thromboembolism in relatives of symptomatic probands with thrombophilia: a systematic review. Thromb Haemost 2003;90:17-26.

5. Wu O, Robertson L, Twaddle S, et al. Screening for thrombophilia in high-risk situations: systematic review and cost-effectiveness analysis. The Thrombosis: Risk and Economic Assessment of Thrombophilia Screening (TREATS) study. Health Technol Assess 2006;10:1-110.

6. Rey E, Kahn SR, David M, Shrier I. Thrombophilic disorders and fetal loss: a meta-analysis. Lancet 2003;361:901-908.

7. Kovalevsky G, Gracia CR, Berlin JA, Sammel MD, Barnhart KT. Evaluation of the association between hereditary thrombophilias and recurrent pregnancy loss: a meta-analysis. Arch Intern Med 2004;64:558-563.

8. Rosendaal FR, Siscovick DS, Schwartz SM, et al. Factor V Leiden (resistance to activated protein $C$ ) increases the risk of myocardial infarction in young women. Blood 1997;89:2817-2821.

9. Grody WW, Griffin JH, Taylor AK, Korf BR, Heit JA. American College of Medical Genetics consensus statement on factor V Leiden mutation testing. Genet Med 2001;3:139-148.

10. Press RD, Bauer KA, Kujovich JL, Heit JA. Clinical utility of factor V Leiden (R506Q) testing for the diagnosis and management of thromboembolic disorders. Arch Pathol Lab Med 2002;126:1304-1318.

11. Spector EB, Grody WW, Matteson CJ, et al. Technical standards and guidelines: venous thromboembolism (factor V Leiden and prothrombin 20210G $>$ A testing): a disease-specific supplement to the standards and guidelines for clinical genetics laboratories. Genet Med 2005;7:444-453.

12. Deep Vein Thrombosis Treatment Guideline. DP-1036. Group Health Cooperative, 2002.

13. Deep Vein Thrombosis Treatment Guideline. DP-1036. Group Health Cooperative, 2003 
14. Gene Tests website. Available at: http://www.genetests.org/.

15. Rodger MA, Carrier M, Keely E, et al. The management of thrombophilia during pregnancy: a Canadian survey. J Obstet Gynaecol Can 2002;24:946952.

16. Grimshaw JM, Thomas RE, MacLennan G, et al. Effectiveness and efficiency of guideline dissemination and implementation strategies. Health Technol Assess 2004;8:iii-iv, 1-72.

17. John BJ, Irukulla S, Mendall MA, Abulafi AM. Do guidelines improve clinical practice?-A National Survey on Surveillance Colonoscopies. [published online ahead of print April 10, 2009.] Colorectal Dis. doi://10.111/ j.1463-1318.2009.01869.x.
18. Riegert-Johnson DL, Macaya D, Hefferon TW, Boardman LA. The incidence of duplicate genetic testing. Genet Med 2008;10:114-116

19. Hull RD, Pineo GF, Brant RF, et al. Self-managed long-term low-molecular-weight heparin therapy: the balance of benefits and harms. Am J Med 2007;120:72-82

20. Burke W, Psaty BM. Personalized medicine in the era of genomics. JAMA 2007;298:1682-1684

21. Morgan MA, Driscoll DA, Mennuti MT, Schulkin J. Practice patterns of obstetrician-gynecologists regarding preconception and prenatal screening for cystic fibrosis. Genet Med 2004;6:450-455.

22. Driscoll DA, Morgan MA, Schulkin J. Screening for Down syndrome: changing practice of obstetricians. Am J Obstet Gynecol 2009;200: 459.e1-e9. 